\title{
Análisis psicométrico de una escala para medir el miedo al delito en jóvenes ecuatorianos
}

\author{
Hiram Reyes-Sosa* y Verónica Molina-Coloma \\ Universidad del País Vasco, San Sebastián, España
}

Recibido, febrero 16/2017

Concepto de evaluación, abril 28/2017

Aceptado, julio 15/2017
Referencia: Reyes-Sosa, H. \& Molina-Coloma, V. (2018). Análisis psicométrico de una escala para medir el miedo al delito en jóvenes ecuatorianos. Acta colombiana de Psicología, 21(1), 290-299. doi: http://www.dx.doi. org/10.14718/ACP.2018.21.1.13

Resumen

En Ecuador, al igual que en el resto de Latinoamérica, la problemática de la inseguridad es innegable, y a pesar de que en el país existen encuestas que miden la percepción de inseguridad o la victimización, no existe ningún instrumento que mida el miedo al delito. La presente investigación tiene como propósito realizar un estudio psicométrico de una escala del miedo al delito que parte de una perspectiva psicosocial. Para validar este modelo teórico se realizó un análisis factorial exploratorio (AFE) y un análisis factorial confirmatorio (AFC) con una muestra conformada por 298 estudiantes universitarios de la ciudad de Ambato, Ecuador, con un promedio de edad de 21.28 años $(\mathrm{DT}=1.62)$. Los resultados muestran que la escala presenta índices de bondad de ajuste satisfactorios. Específicamente, se utilizaron dos modelos explicativos del miedo al delito, uno de primer orden y otro de segundo orden, siendo este último el que explicó mejor la estructura psicosocial del miedo al delito. Palabras claves: Miedo al delito, análisis factorial confirmatorio, fiabilidad, inseguridad, modelos de ecuaciones estructurales.

\section{Psychometric analysis of a scale to measure fear of crime in Ecuadorian youths}

\begin{abstract}
In Ecuador, as in the rest of Latin America, the problem of insecurity is undeniable. Although there are surveys in the country that measure the perception of insecurity or victimization, there is no instrument that measures fear of crime. This research was aimed at the structural validation of a scale to measure the fear of crime from the psychosocial perspective. To validate this theoretical model, an exploratory factor analysis (EFA) and confirmatory factor analyses (CFA) were conducted. A total of 298 students from the city of Ambato, Ecuador, participated in the study. The average age was 21.28 years $(\mathrm{SD}=1.62)$. Results showed satisfactory psychometric and structural properties of the scale. Furthermore, two theoretical models of fear of crime were developed: a first order factor and a second order factor. Results confirm the relevance of the second order model for explaining the psychosocial construction of the fear of crime.

Key words: Fear of crime, confirmatory factor analysis, reliability, insecurity, structural equations model.
\end{abstract}

* Doctor en Psicología Social, Universidad del País Vasco / Euskal Herriko Unibertsitatea, Avda. de Tolosa, 70, 20018, Donostia, España. Tel.: 005216672013788. hiramreyez@gmail.com 


\title{
Análise psicométrica de uma escala para medir o medo do delito em jovens equatorianos
}

\begin{abstract}
Resumo
No Equador, assim como em toda a América Latina, a problemática da insegurança é inegável e, apesar de existirem, no país, pesquisas que meçam a percepção de insegurança ou vitimização, não há nenhum instrumento que meça o medo do delito. A presente pesquisa tem como propósito realizar um estudo psicométrico de uma escala do medo do delito que parte de uma perspectiva psicossocial. Para validar esse modelo teórico, realizou-se uma análise fatorial exploratória (AFE) e uma análise fatorial confirmatória (AFC) com uma amostra conformada por 298 estudantes universitários da cidade de Ambato, Equador, com uma média de idade de 21.28 anos $(\mathrm{DT}=1.62)$; os resultados mostraram que a escala apresenta índices de bondade de ajuste satisfatórios. Especificamente, utilizaram-se dois modelos explicativos do medo do delito, um de primeira ordem e outro de segunda ordem; este último explicou melhor a estrutura psicossocial do medo do delito.

Palavras-chave: Análise fatorial confirmatória, confiabilidade, insegurança, medo do delito, modelos de equações estruturais.
\end{abstract}

\section{INTRODUCCIÓN}

En los últimos años, Ecuador ha sido considerado como uno de los principales países en Latinoamérica gracias a su amplio desarrollo económico y social (Latinobarómetro, 2013). Sin embargo, al igual que en el resto de Latinoamérica, la problemática de la inseguridad es innegable. Particularmente, durante la década de los 90, la inseguridad presentó sus primeras consecuencias sociales en el país, pues la frecuencia de los delitos creció exponencialmente; por ejemplo, los secuestros aumentaron un $60 \%$, las extorsiones un $106 \%$ y los homicidios se duplicaron; $y$, en concreto, se presentaron de 7 a 14 casos anuales por cada 100 mil habitantes (Jarrín, 2005); teniendo esto en cuenta, en el año de 1999 el sistema de gobierno ecuatoriano reorganizó toda la estructura judicial con la finalidad de controlar dicho fenómeno social (Ojeda, 2010); pero, pese a esta iniciativa -según los datos de la policía judicial- los índices delictivos entre los años 2000 y 2009 no tuvieron una reducción significativa, y, por el contrario, los índices de homicidio presentaron nuevamente un incremento significativo (Centro Ecuatoriano de Análisis de Seguridad Integral, 2013).

Dicha restructuración judicial, sumada a su precaria experticia, dio como resultado que se desarrollara y estableciera un clima de inseguridad en el país caracterizado por altos índices delictivos. En este sentido, es comprensible que para el año 2013 -teniendo en cuenta los datos del Latinobarómetro (2013)-, el $31 \%$ de los ciudadanos ecuatorianos indicaron que el principal problema del país era la inseguridad y el $49 \%$ que sentía preocupación por llegar a ser víctima de un delito. Estos datos permiten evidenciar la importancia de aproximarse al estudio de las problemáticas sobre la inseguridad; particularmente, las problemáticas relacionadas con el miedo al delito.

Por otra parte, a pesar de que en Ecuador existen encuestas que miden la percepción de inseguridad o la victimización, cabe señalar que no existe ningún instrumento que mida el miedo al delito (Gallardo-León, 2009; Molina-Coloma, Reyes-Sosa \& Larrañaga, 2015). Por ello, y dada la gran relevancia social de esta problemática en el contexto ecuatoriano, es que resulta de gran importancia construir medidas para operacionalizar este fenómeno.

Por último -y con un interés teórico-, si bien existe una amplia tradición en las investigaciones del miedo al delito que se aproximan a conocer sus causas (Farrall, Jackson \& Gray, 2009), también es cierto que en la literatura no existen un consenso con respecto a la operacionalización de este constructo (Gabriel \& Grave, 2003). En este sentido, a continuación se hace un acercamiento a la teoría del miedo al delito, su operacionalización y su medición.

\section{Miedo al delito, ¿un concepto monolítico?: una visión} psicosocial para su operacionalización

La inseguridad y el miedo asociado a la delincuencia han sido parte de las preocupaciones de los seres humanos desde hace ya bastante tiempo (Fernández \& Grijalva, 2012). Sin embargo, fue hasta la década de 1970 que el miedo al delito se convirtió en un tema de gran relevancia social (Gabriel \& Greve, 2003; Lee, 2007; Warr, 2006), aunque en sus inicios era medido con un solo ítem -lo que se denomina "medida global"-, donde la pregunta planteada en aras de investigación era " ¿se siente seguro usted caminando por su vecindario de noche?" (Hale, 1996; Warr, 2006).

No obstante, diversos autores han señalado que una sola medida es imprecisa, que no proporciona información de calidad para comprender este fenómeno (Vozmediano, San Juan \& Vergara, 2008), y que este tipo de preguntas causan un sesgo, puesto que provocan una respuesta de sobreevaluación del riesgo por parte de los sujetos. De esta manera, una única medida puede ser considerada como imprecisa y no refleja la complejidad de las dimensiones que pueden explicar el miedo al delito (Fernández \& Grijalva, 2012). 
Partiendo de un supuesto monolítico de medida, Hale (1996) señala que son múltiples los estudios que han intentado aportar evidencia de los factores que pueden potencializar el miedo, y que algunas investigaciones han centrado su interés en la medida global (Lee, 2007; Winkel, 1999), en las fracturas del sentimiento de pertenencia a la comunidad (Sampson \& Stephen, 1999, Sutherland, 1939), el abandono de los espacios públicos (Doran \& Brian, 2005; Moore \& Shepherd, 2007; Williamson, David \& Richard, 2006), las emociones como promotoras del miedo (Frijda, Manstead \& Bem, 2000; Loewenstein, Weber, Hsee \& Welch, 2001; Slovic, Finucane, Peters \& Macgregor, 2004) o en medidas más orientadas al estudio de la percepción de riesgo de victimización (Garofalo, 1979). Todas estas investigaciones han intentado dar una mejor explicación a este fenómeno de formas multivariantes de apropiación y, por tanto, demuestran la complejidad del estudio de esta problemática, así como su falta de consenso y operacionalización (Vozmediano et al., 2008).

De este modo, las investigaciones desde la visión monolítica posibilitaron que diversos autores señalaran la dificultad que presenta el miedo al delito en su medición (Hale, 1996; Jackson, 2005), y que, partiendo de una concepción polisémica, se empezaran a construir medidas más complejas para operacionalizar dicho constructo (Killias, 1990; Van der Wurff, Van Staalduinen \& Stringer, 1989). Desde esta perspectiva, autores como Van der Wurff et al. (1989) proponen una medida integral para el estudio del miedo al delito denomina Modelos de Psicología Social, donde proponen cuatro componentes sociales que deben ser medidos conjuntamente: la atractividad, la intencionalidad criminal, el poder y la inestabilidad contextual.

Por su parte, Fattah y Sacco (1989) se aproximan al estudio de miedo al delito argumentando que el mismo es un constructo con dos características principales: una emocional y otra psicológica. Y, a partir de esto, desarrollaron un modelo para el estudio del miedo que centra su interés en tres aspectos que se deben medir conjuntamente: el sentimiento de inseguridad, la preocupación por el delito y la percepción de riesgo.

Asimismo, el trabajo desarrollado por Killias (1990)desde la visión de la vulnerabilidad-puede ser considerado como pionero en la medición psicosocial de miedo al delito (Hale, 1996; Jackson, 2009), pues propone que el miedo al delito debe ser operacionalizado con la definición de la exposición de riesgo, la pérdida de control y la afección de las consecuencias del delito. Este modelo es especialmente relevante porque incorpora la noción del contexto social y el componente situacional, y permite identificar los procesos que potencian o disminuyen el miedo al delito -como los son, por ejemplo, las habilidades físicas-.
Estos estudios permitieron introducir la noción psicosocial en el estudio de miedo al delito (Jackson, 2009) y, por tanto, dieron cabida a estudios como el de Gabriel y Grave (2003), quienes argumentan que dicho constructo debe ser comprendido tanto a nivel individual como social, de modo que no solo los factores individuales sean los que promuevan en mayor medida el miedo, sino que los factores sociales - o contextuales - también ejercen un papel importante en este fenómeno (Gabriel \& Grave, 2003).

Teniendo esto en cuenta, el miedo al delito debe ser concebido como un estado transitorio -el grado de miedo que genera la posibilidad de ser víctima de un delito (contexto), asociado a factores sociales (estado)- o como una predisposición-la probabilidad de tener experiencias previas (victimización), asociadas a factores individuales (predisposición) que promueven el miedo-(Gabriel \& Grave, 2003).

Más recientemente, Jackson $(2005,2006,2009)$ plantea que en los estudios del miedo al delito no solo se debe hacer la pregunta sobre por qué algunas personas se preocupan más que otras, sino que deben definirse los mecanismos y la percepción subjetiva que se tiene sobre el delito (Gabriel \& Greve, 2003; Jackson, 2009). De este modo, los estudios del miedo al delito deben definir tanto la ansiedad en espacios públicos como la preocupación por el delito; esto con el fin de establecer si la ansiedad frente al delito es uno de los riesgos que aumenta la probabilidad de ser víctima o no de un delito, y si el hecho de no controlar el evento ni las consecuencias del mismo pueden provocar un aumento en la preocupación de ser víctima (Killias, 1990).

En este sentido, partiendo de la noción de la vulnerabilidad y haciendo un esfuerzo por solucionar el problema de la evaluación no monolítica del miedo al delito, Jackson (2009) desarrolla una escala para medir el miedo al delito desde una visión psicosocial, la cual centra su atención en cuatro dimensiones principales operacionalizadas conjuntamente: la preocupación acerca del delito, la percepción de probabilidad del delito, la percepción de control del delito y la percepción de las consecuencias del delito. Además, estas cuatro dimensiones se aplican a cuatro contextos delictivos distintos, por ejemplo, ser atracado por un desconocido en la calle o ser amenazado en la calle (Jackson, 2009). Específicamente, el componente de preocupación de la escala mide el aspecto emocional, mientras que los componentes de probabilidad, control y consecuencias miden el riesgo de victimización, es decir, el aspecto del riesgo percibido (noción de vulnerabilidad).

Teniendo todo lo anterior en cuenta, el presente estudio tiene como objetivo central la adaptación y análisis psicométrico de la escala de miedo al delito desarrollada por Jackson (2009) -replicada en México por Reyes, Valencia y Larrañaga (2015)-en el contexto ecuatoriano, y establecer 
la fiabilidad y validez del instrumento al comprobar su estructura interna y su ajuste al modelo teórico cuatridimensional propuesto por Jackson (2009).

\section{MÉTODO}

\section{Muestra}

El presente es un estudio transversal llevado a cabo con una muestra -seleccionada de manera no probabilística e incidental- de 298 jóvenes (142 hombres y 156 mujeres) que se encontraban estudiando en la Universidad Técnica de Ambato (UTA), que presentaban una media de edad de 21.28 años $(\mathrm{DT}=1.62)$, y pertenecían a diferentes centros universitarios -como psicología, terapia física, contabilidad y auditoría e ingeniería en sistemas-.

\section{Instrumento}

Se utilizó la Escala de miedo al delito (Jackson, 2009) en su versión adaptada al español (Reyes et al., 2015), compuesta por 16 ítems que se agrupan en cuatro dimensiones - preocupación acerca del delito, percepción de probabilidad del delito, percepción de control del delito y percepción de las consecuencias del delito- evaluadas a partir de cuatro contextos delictivos: ser atracado por un desconocido en la calle, ser acosado o amenazado en la calle, ser robado de manera no violenta y que alguien irrumpa en mi casa mientras mis parientes están en ella. De este modo, cada dimensión es combinada con cada contexto delictivo para crear un modelo 4 x 4; y las preguntas formuladas, según cada dimensión, son las siguientes:

- Preocupación: “¿En qué medida se ha sentido preocupado por ello?", cuyo intervalo de respuesta es: 1 = ni una vez durante el último mes, 2 = una o dos veces durante el último mes, 3 = una o dos veces durante la semana pasada y $4=$ todos los dias.

- Probabilidad: “¿En qué medida cree que le puede ocurrir eso a usted?", cuyo intervalo de respuesta va de $1=$ no me ocurrirá en absoluto a $5=$ seguro que me ocurrirá.

- Control: “¿En qué medida se siente capaz de controlar el llegar a ser víctima de un delito?", cuyo intervalo de respuesta va de $1=$ en absoluto no me siento capaz a 5 = totalmente seguro que controlo.

- Consecuencias: "¿En qué medida piensa que una experiencia de este tipo puede afectar a su vida?", cuyo intervalo de respuesta va de $1=$ nada en absoluto a $5=$ me afectaría mucho.

En la versión en español (Reyes et al., 2015), el coeficiente alfa de la escala total fue de .86 , y en las puntuaciones de cada dimensión se obtuvieron puntajes superiores a .78, a saber: preocupación $(\alpha=.83)$, probabilidad $(\alpha=.78)$, control $(\alpha=.78)$ y consecuencias $(\alpha=.82)$.

\section{Procedimiento}

Con el objetivo de detectar los problemas de redacción y compresión de los ítems, en la presente investigación se realizó un muestreo piloto, desarrollado por colaboradores debidamente entrenados (American Psychological Association, 1999; Medrano \& Trógolo, 2014). Posteriormente, teniendo en cuenta las correcciones correspondientes, se preparó el instrumento final para su pasación definitiva.

Tras la presentación de los objetivos y alcances de esta investigación tanto a los directivos como a los alumnos, donde se obtuvo el consentimiento de las autoridades para la aplicación del instrumento, se procedió a la aplicación la escala, la cual contó, además, con un formato de consentimiento informado para cada estudiante, donde se establecía que todas las respuestas serían anónimas.

\section{Análisis de los datos}

Para validar la adaptación de la escala, primero que todo, se obtuvieron datos descriptivos de cada ítem, índice de homogeneidad y los alfas de Cronbach para la escala total y para cada dimensión. Posteriormente, con el uso del programa SPSS 22, se realizó el análisis factorial exploratorio (AFE) para estudiar la estructura factorial de la escala, el análisis discriminante en función de sexo y los tamaños de efecto; $y$, teniendo en cuenta que el objetivo de este artículo es validar un instrumento, se realizó el análisis factorial confirmatorio con el programa M-PLUS 7.1.

Adicionalmente, se hizo uso de diversos criterios para confirmar la estructura de la escala. Por ejemplo, se utilizó el estadístico Chi-cuadrado $\left(X^{2}\right)$, que permite probar el modelo nulo frente al hipotetizado o propuesto - un puntaje no estadísticamente significativo $(p>.05)$ puede interpretarse como indicador de un adecuado ajuste del modelo (Miranda-Zapata, Riquelme-Mella, Cifuentes-Cid \& Riquelme-Bravo, 2014)-, donde se considera que un Chi-cuadrado dividido por los grados de libertad de alrededor de tres es satisfactorio, ya que este se encuentra relacionado con el tamaño muestral y que generalmente tiende a presentar diferencias en muestras grandes.

Por otra parte, la evaluación de la bondad del ajuste de los datos se determinó utilizando como índice de ajuste incremental el CFI (Comparative Fit Index) y el TLI (Tucker-Lewis Index); y, como índices de ajuste absoluto, se utilizó el RMSEA(Root Mean Square Error Aproximation) y el SRMR (Root Mean Square Residual). El mejor ajuste del modelo a los datos se presenta cuando los valores son menores en el $\chi 2$, SRMR $(<.08)$ y RMSEA $(<.07)$, y mayores el CFI y el TLI (> .90) (Chen, 2007; Steiger, 2007). 


\section{RESULTADOS}

En general, con los análisis realizados, se obtuvo la fiabilidad interna para la escala total del miedo al delito, pues se establece la ausencia de errores de medición en el instrumento (Virla, 2010). Específicamente, la fiabilidad de la escala global como constructo obtuvo una consistencia interna alta (.89) (Santisteban, 2009), y con respecto al índice de homogeneidad corregida para cada ítem (véase Tabla 1), índice que permite determinar el grado en que los ítems de la escala están agrupados en un solo factor (Virla, 2010), se obtuvo que la mayoría de los ítems presentaron índices satisfactorios superiores a valores de .30, excepto el ítem 12 -que presentó un índice de .29-, sin embargo, se decidió mantener este ítem debido a que se aproxima al límite propuesto y que aporta información teórica sobre el control del delito (Magnusson, 1978; Martínez, 1995).

Análisis factorial exploratorio de la escala del miedo al delito

En el AFE, los resultados de la prueba de esfericidad de $\operatorname{Bartlett}\left(X^{2}=1793.756, g l=120, p<.000\right)$ mostraron que los ítems eran dependientes; y el índice Kaiser-Meyer-Olkin (.81) indicó una buena adecuación muestral y una buena correlación entre los ítems, lo que dio cuenta de que los ítems son adecuados para la aplicación del análisis factorial (Hambleton \& Patsula, 1999). Específicamente, se utilizó el método de extracción componentes principales y rotación Varimax, ya que se buscaba reducir el número de variables perdiendo la menor información posible y manteniendo la ortogonalidad entre las dimensiones (Santisteban, 2009); finalmente, el análisis de componentes principales arrojó cuatro factores que explicaron el $60.5 \%$ de la varianza.

En concreto, el Factor 1 -dimensión que enfatiza la preocupación por llegar a ser víctima de un delito-explicó el $31 \%$ de la varianza y agrupó los ítems 1 (.79), 2 (.69), 3(.78) y 4 (.73); el Factor 2 -dimensión que enfatiza la probabilidad de ser víctima de un delito- explicó el $13 \%$ de la varianza y agrupó los ítems 5 (.64), 6 (.67), 7 (.71) y 8 (.70); mientras que el Factor 3 -dimensión que enfatiza el sentimiento de control por llegar a ser víctima de un delito-explicó el $9.7 \%$ de la varianza y agrupó los ítems $9(.74), 10(.73), 11(.76)$ y 12 (.68); y el Factor 4 -dimensión que enfatiza la afección que un delito puede tener en la vida cotidiana- explicó el

Tabla 1

Estadísticos para cada item de la Escala de Miedo al Delito

\begin{tabular}{|c|c|c|c|c|}
\hline Reactivos & $\mathrm{M}$ & DT & $\mathrm{IHC}$ & Alfe \\
\hline \multicolumn{5}{|l|}{ Preocupación acerca del delito } \\
\hline 1. Ser atracado por un desconocido en la calle. & 2.34 & 1.10 & .525 & .746 \\
\hline 2. Ser acosado o amenazado en la calle. & 2.03 & 1.05 & .534 & .741 \\
\hline 3. Ser robado de manera no violenta. & 2.11 & 1.10 & .540 & .744 \\
\hline 4. Que alguien irrumpa en mi casa mientras mis parientes están en ella. & 1.67 & .93 & .441 & .751 \\
\hline \multicolumn{5}{|l|}{ Percepción de probabilidad del delito } \\
\hline 5. Ser atracado por un desconocido en la calle. & 3.13 & .85 & .401 & .747 \\
\hline 6. Ser acosado o amenazado en la calle. & 2.84 & .90 & .468 & .744 \\
\hline 7. Ser robado de manera no violenta. & 3.16 & .96 & .374 & .748 \\
\hline 8. Que alguien irrumpa en mi casa mientras mis parientes están en ella. & 2.69 & .95 & .347 & .753 \\
\hline \multicolumn{5}{|l|}{ Percepción de control del delito } \\
\hline 9. Ser atracado por un desconocido en la calle. & 2.92 & 1.00 & .383 & .789 \\
\hline 10. Ser acosado o amenazado en la calle. & 3.08 & .97 & .316 & .783 \\
\hline 11. Ser robado de manera no violenta. & 3.09 & .98 & .374 & .788 \\
\hline 12. Que alguien irrumpa en mi casa mientras mis parientes están en ella. & 2.91 & 1.03 & .289 & .780 \\
\hline \multicolumn{5}{|l|}{ Percepción de las consecuencias del delito } \\
\hline 13. Ser atracado por un desconocido en la calle. & 3.18 & 1.05 & .611 & .742 \\
\hline 14. Ser acosado o amenazado en la calle. & 3.09 & 1.07 & .629 & .742 \\
\hline 15. Ser robado de manera no violenta. & 2.91 & 1.08 & .520 & .742 \\
\hline 16. Que alguien irrumpa en mi casa mientras mis parientes están en ella. & 3.31 & 1.13 & .403 & .751 \\
\hline
\end{tabular}

Nota. M = Media; DT = Desviación típica; $\mathrm{IHC}$ = índice de homogeneidad corregida; Alfa = alfa si se elimina el ítem. 
$6.8 \%$ de la varianza y agrupó los ítems 13 (.62), 14 (.59), $15(.57)$ y $16(.74)$.

Adicionalmente, se calculó el índice de fiabilidad interno a través del alfa de Cronbach en cada dimensión, y las puntuaciones arrojadas fueron: preocupación $=.81$, probabilidad $=.69$, control $=.73$ y consecuencias $=.83$.

Consistencia interna de la escala de miedo al delito: análisis factorial confirmatorio

Modelo de un único factor general. Con la finalidad de confirmar la estructura interna de la escala y demostrar que el constructo de miedo al delito no es monolítico, se planteó un primer modelo que defiende la existencia de un único factor de primer orden -una visión unidimensional del miedo-, y el AFC aplicado para este modelo (Modelo 1) demuestra un ajuste bastante pobre, como era de esperarse $\left(X^{2}=1681.320, g l=105, X^{2} / g l=16.01\right.$; RMSEA $=.184$, $\mathrm{SRMR}=.159,90 \% \mathrm{CI}[.176, .192] ; \mathrm{CFI}=.441 ; \mathrm{TLI}=.361)$.
Modelo confirmatorio de primer orden. Posteriormente, con la intención de confirmar la estructura cuatridimensional del miedo al delito, se realizó un modelo de primer orden (Modelo 2) basado en la estructura teórica propuesta por Jackson $(2005,2006,2009)$ en estudios previos; y el AFC para este modelo (véase Figura 1) presentó ajustes satisfactorios $\left(X^{2}=241.823, g l=96, p<.000 ; X^{2} / g l=2.51\right.$; RMSEA $=.070$, SRMR $=.052,90 \%$ CI $[.060, .081]$; $\mathrm{CFI}=.920 ; \mathrm{TLI}=.901)$.

Modelo confirmatorio de segundo orden. Una vez confirmado el modelo cuatridimensional propuesto por Jackson (2009) y con el interés teórico de aportar evidencia de que el miedo al delito es un constructo unificador de segundo orden (variable latente exógena) operacionalizado por cuatro dimensiones, se realizó un tercer modelo (Modelo 3), cuyo análisis (véase Figura 2) arrojó ajustes aceptables $\left(\chi^{2}=259.270, g l=98, p<.000 ; \chi^{2} / g l=2.64\right.$;

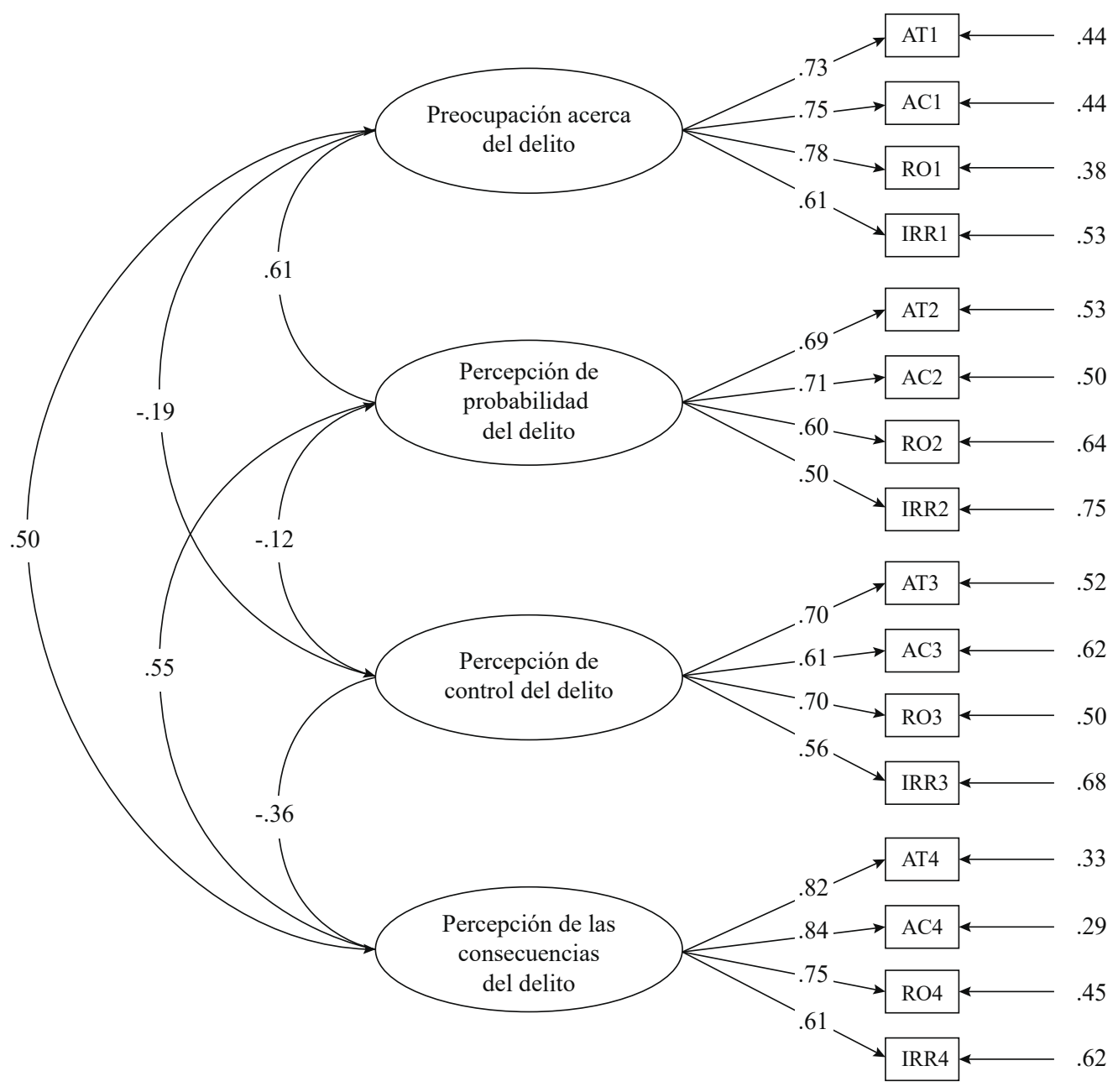

Figura 1. Análisis factorial confirmatorio de primer orden del miedo al delito. 
RMSEA $=.072$, SRMR $=.054,90 \%$ CI $[.063, .083]$; $\mathrm{CFI}=.910 ; \mathrm{TLI}=.895)$.

A pesar de que este modelo presentó menor parsimoniosidad en su ajuste en comparación con el Modelo 2, los resultados permiten aportar evidencia teórica con respecto a que el miedo al delito es un constructo polisémico (Jackson, 2009). En este sentido, el miedo al delito se posiciona como un factor de segundo orden, y las dimensiones de la preocupación acerca del delito (.66), la percepción de probabilidad del delito (.87), la percepción de control del delito (-.29) y la percepción de las consecuencias del delito $(.80)$ se posicionan como factores de primer orden (variables latentes endógenas) (Fernández \& Grijalva, 2012; Fernández, López \& Mariel, 2005; Ruiz, Pardo \& San Martín, 2010).

\section{Validez discriminante}

Por último, con el objetivo de obtener datos adicionales para la validez externa de la escala, adicionalmente se examinaron las diferencias entre los componentes de miedo al delito en función del sexo, se aplicaron análisis de varianza para comprobar las diferencias de medias, y se estimaron los tamaños del efecto con el estadístico eta cuadrado.

Al respecto, cabe señalar que a nivel global $\left[F_{(1.297)}=10.41\right.$; $p=.000, \eta 2=.139]$ y en cada variable de estudio se presentaron diferencias estadísticamente significativas entre hombres y mujeres (véase Tabla 2), puesto que las mujeres obtuvieron calificaciones más altas que los hombres en las variables de preocupación acerca del delito, probabilidad del delito y consecuencias del delito; mientras que los hombres obtuvieron puntuaciones más altas en la variable de control del delito.

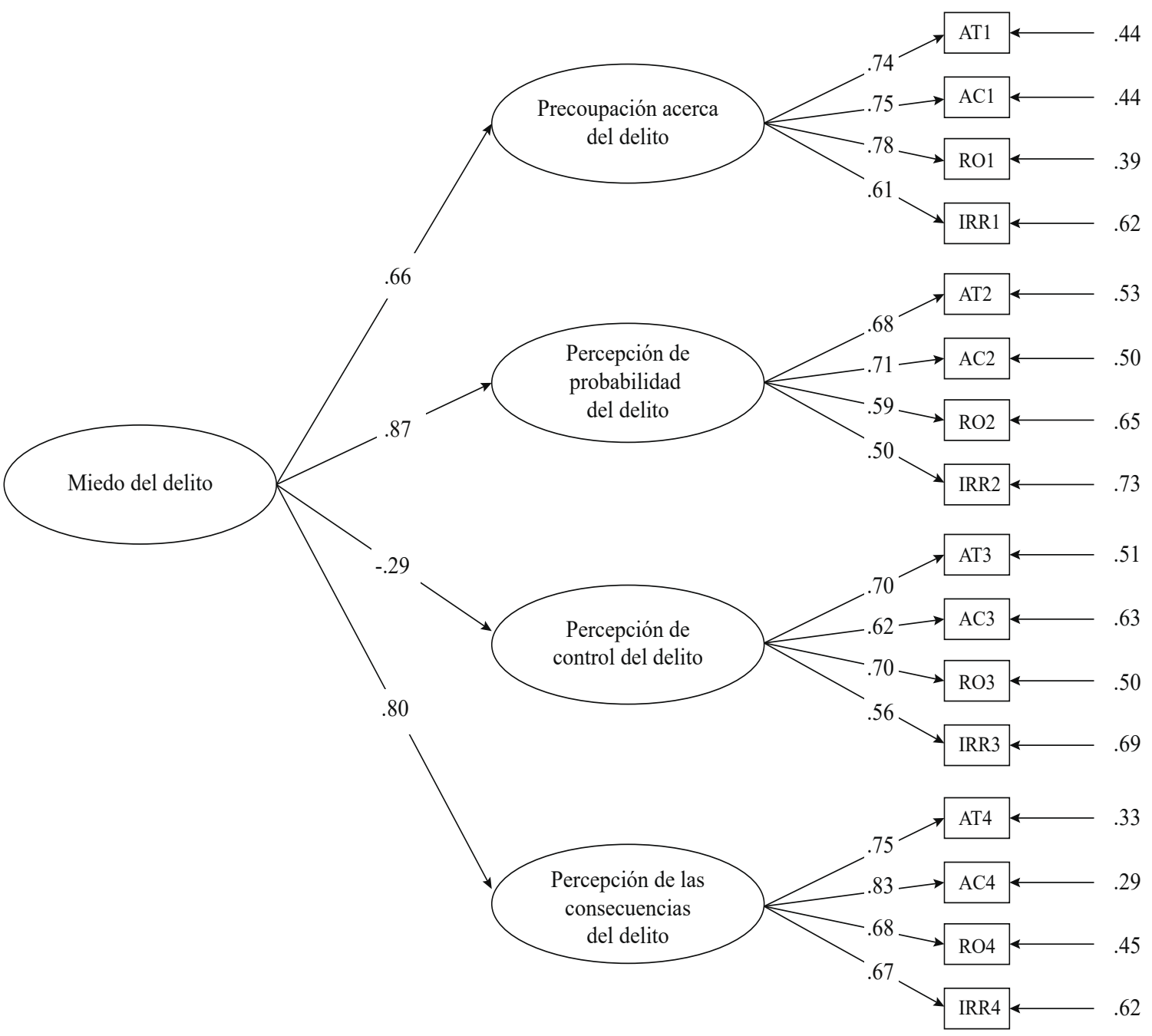

Figura 2. Análisis factorial confirmatorio de segundo orden del miedo al delito. 
Tabla 2

Comparación hombres y mujeres para las variables preocupación, probabilidad, control, consecuencias

\begin{tabular}{cccccc}
\hline & \multicolumn{5}{c}{ Género } \\
\cline { 2 - 6 } & $\begin{array}{c}\text { Hombres } \\
\mathrm{n}=142\end{array}$ & $\begin{array}{c}\text { Mujeres } \\
\mathrm{n}=156\end{array}$ & $\mathrm{~F}$ & $\mathrm{P}$ & $\eta 2$ \\
\cline { 2 - 6 } & $\mathrm{M}(\mathrm{DT})$ & $\mathrm{M}(\mathrm{DT})$ & .000 & .048 \\
\hline Preocupación & $1.80(.75)$ & $2.22(.87)$ & 18.32 & .000 & .072 \\
Probabilidad & $2.80(.66)$ & $3.11(.64)$ & 17.17 & .000 & .047 \\
Control & $3.22(.71)$ & $2.80(.73)$ & 24.59 & .000 & .103 \\
\hline Consecuencias & $2.80(.88)$ & $3.43(.79)$ & 42.82 & & \\
\hline
\end{tabular}

\section{DISCUSIÓN}

En lo que concierne a la estructura y fiabilidad de la escala, se debe señalar que el modelo nulo (Modelo 1) presentó un pobre ajuste, como era de esperarse; lo que permite dar cuenta de que el constructo de miedo al delito no es un concepto monosémico, sino polisémico. Por tal razón, el índice de ajuste comparado (CFI) para el modelo 2 y 3 resultó ser mayor a .90 y el Chi-cuadrado dividió por los grados de libertad fue inferior a 3 .

De esta manera, los 16 ítems se distribuyeron en las cuatro dimensiones esperadas: preocupación acerca del delito, percepción de probabilidad del delito, percepción de control del delito y percepción de las consecuencias del delito, tal como se encontró en Jackson (2009). Además, el error cuadrático medio de aproximación (RMSEA) para ambos modelos se fue menor o igual a .07, lo que indica, nuevamente, un buen ajuste (Steiger, 2007).

Por otra parte, se debe señalar que a pesar de que los modelos 2 y 3 presentan índices de ajuste similares, dado que los modelos no están anidados (criterio empírico), los ajustes estadísticos no son indicadores plausibles que permitan evidenciar cuál de los dos modelos explica idóneamente el miedo al delito; a pesar de que el Modelo 3 es preferible por su sencillez y porque tiene más grados de libertad (2), lo que lleva a un incremento significativo del Chi-cuadrado (Fujikoshi, 2000). Sin embargo, haciendo énfasis en el posicionamiento-criterio teórico, y dado que los datos obtenidos son indicadores de la bondad de ajuste de los modelos de esta muestra en concreto, el Modelo 3 explica mejor la construcción psicosocial del concepto del miedo al delito, tal como fue desarrollado desde Van der Wurff et al. (1989) hasta la actualidad (Jackson, 2009; Reyes et al., 2015).
Adicionalmente, el hecho de que las dimensiones se agrupen en un factor de segundo orden permite confirmar que el miedo al delito es un concepto polisémico que se explica a través de las cuatro dimensiones previamente mencionadas; sin embargo, es importante recalcar que la emoción misma del miedo, que puede ser personal o percibida en el contexto, no hace parte -de manera directa- del instrumento -aunque teóricamente se ha considerado que el componente de preocupación es el afectivo-; y, en ese sentido, sería interesante evaluar también el afecto mismo del miedo dentro de la escala, y comparar este instrumentos con escalas de clima emocional que incluyan la evaluación del miedo y la inseguridad (De Rivera \& Páez, 2007).

Por otra parte, en lo que concierne a la discriminación de la escala, estudios previos han corroborado lo encontrado en esta investigación (Fox, Nobles \& Piquero, 2009); por lo que se corrobora que el grupo de mujeres es el que presenta mayores niveles de miedo, en comparación con el grupo de hombres. De igual forma, el tamaño del efecto también es relevante, puesto que es un indicador de validez de la escala; así, si las mujeres presentan mayores niveles de miedo, esto se debe a que se preocupan en mayor medida, perciben que tienen mayor probabilidad de ser víctimas de un delito, experimentan menor sensación de control y perciben que las consecuencias del delito pueden afectar en mayor medida a su grupo de pertenencia (Jackson, 2009; Scott, 2003).

Finalmente, cabe señalar que si bien la escala adaptada a Ecuador ha sido eficaz para medir el miedo al delito, también se debe cuestionar cuáles son los factores que promueven en mayor medida este fenómeno. Desde la perspectiva inglesa, Jackson (2009) propone que las habilidades físicas y los juicios valorativos son los procesos que mayormente promueven el miedo al delito en contextos anglosajones. Sin embargo, estudios previos en México (Vilalta, 2009a, Vilalta, 2009b; 
Reyes et al., 2015), han demostrado que la victimización y la inestabilidad contextual (inseguridad) son dos factores que promueven en mayor medida el miedo al delito en contextos latinoamericanos. En el caso de Ecuador, con el presente estudio - llevado a cabo en Ambato-, es posible evidenciar, de igual forma, lo expuesto anteriormente: la victimización promueve en mayor medida la preocupación por el miedo al delito (Molina-Coloma et al., 2015).

En suma, a pesar de que en Ecuador se crearon e implementaron mejores herramientas para reducir la inseguridad (por ejemplo, la creación del número de emergencia ecu-911, los botones de pánico en taxis y autobuses) en algunas zonas del país la percepción del miedo al delito permanece. Y esto corrobora lo encontrado previamente con respecto a que el miedo resulta ser tan significativo como el delito mismo (Föhrig, 2006), y que un mayor miedo al delito -especialmente en las víctimas- provoca cambios en las rutinas cotidianas (Kanan \& Pruitt, 2002), como lo es el evitar salir a lugares o zonas específicas consideradas como peligrosas (Carrión, 1994).

Teniendo lo anterior en cuenta, se concluye que el instrumento evaluado en este estudio puede ser una medida adecuada para comprender la problemática del miedo al delito, $y$, por tanto, puede ser utilizado en el futuro para que se desarrollen estrategias que permitan disminuir la desconfianza social y, con ello, promover la sana convivencia y el bienestar de la sociedad.

\section{REFERENCIAS}

American Psychological Association. (1999). Standards for psychological and educational tests. Washington, D.C.: Autor.

Carrión, F. (1994). Violencia Urbana e Inseguridad Ciudadana: Ecuador. En PNUD/Plan Nacional de Rehabilitación Programa Residencial (Ed.), Memorias del Seminario Internacional "Violencia Urbana e Inseguridad Ciudadana" (pp. 163-168). Bogotá: PNUD/Plan Nacional de Rehabilitación Programa Residencial.

Centro Ecuatoriano de Análisis de Seguridad Integral y Comisión de Estadística de Seguridad Integral. (2013). Estadísticas de seguridad integral noviembre 2013. Recuperado de http://www.ant.gob.ec/index.php/noticias/estadisticas-deseguridad-integral-2013.

Chen, F. F. (2007) Sensitivity of Goodness of Fit Indexes to Lack of Measurement Invariance. Structural Equation Modeling: A Multidisciplinary Journal, 14(3), 464-504. doi: 10.1080/10705510701301834.

De Rivera, J., \& Páez, D. (2007). Emotional climate, human security and cultura of peace. Journal for Social Issues, 63(2), 233-254.
Doran, B., \& Brian, L. (2005). Investigating the SpatioTemporal Links between Disorder, Crime and the Fear of Crime. The Professional Geographer, 57(1), 1-12. doi: 10.1111/j.0033-0124.2005.00454.x.

Fernández, E., \& Grijalva, A. E. (2012). Diseño y validación de dos escalas para medir el miedo al delito y la confianza en la policía. Revista Española de Investigación Criminológica, 2(10), 1-26.

Fernández, K., López, C., \& Mariel, P. (2005). La satisfacción del consumidor: una aplicación del análisis factorial confirmatorio a la industria automovilística española. Estadística Española, 47(158), 117-141.

Farrall, S., Jackson, J., \& Gray, E. (2009). Social order and the fear of crime in contemporary times. Clarendon Studies in Criminology. Oxford University Press.

Fattah, E. A., \& Sacco, V. F. (1989). Crime and Victimization of the Elderly. Springer-Verlag: New York.

Föhrig, A. (2006). Introducción. En J. Varat \& A. Garland (Eds.), Participación cuidadana y percepción de inseguridad en América Latina (pp. 1-3). Washington: Woodrow Wilson International Center for Scholars.

Fox, K., Nobles, M. R., \& Piquero, A. (2009). Gender, crime victimization and fear of crime. Security Journal, 22(1), 2439. doi: $10.1057 / \mathrm{sj} .2008 .13$.

Frijda, N. H., Manstead, A.S., \& Bem, S. (2000). Emotions and Beliefs: How feelings influence thoughts. Cambridge, Reino Unido: Cambridge University Press.

Fujikoshi, Y. (2000). Transformations with improved chi-squared approximations. Journal of Multivariate Analysis, 72(2), 249-263. doi: https://doi.org/10.1006/ jmva.1999.1854.

Gabriel, U., \& Greve, W. (2003). The psychology of fear of crime: Conceptual and methodological perspectives. British Journal of Criminology, 43(3), 600-614. doi: 10.1093/ bjc/43.3.600.

Gallardo-León, C. (2009). Las Encuestas de victimización en Ecuador. Ciudad Segura, 37, 4-9.

Garofalo, J. (1979). Victimization and fear of crime. Journal of Research in Crime \& Delinquency, 16(1), 80-97. doi: 10.1177/002242787901600107.

Hale, C. (1996). Fear of crime: A review of the literature. International Review of Victimology, 4(2), 79-150.

Hambleton, R. K., \& Patsula, L. (1999). Increasing the validity of adapted tests: Myths to be avoided and guidelines for improving test adaptation practices. Journal of Applied Testing Technology, 1, 1-30.

Jackson, J. (2005). Validating new measures of the fear of crime. International Journal of Social Research Methodology, 8(4), 297-315. doi: http://dx.doi. org/10.1080/13645570500299165.

Jackson, J. (2006). Introducing fear of crime to risk research. Risk Analysis, 26(1), 253-264. doi:10.1111/j.15396924.2006.00715.x. 
Jackson, J. (2009). A Psychological Perspective on Vulnerability in the Fear of Crime. Psychology, crime and law, 15(4), 365-390. doi: 10.1080/10683160802275797.

Jarrín, O. (2005). Politicas Públicas de Seguridad Ciudadana y Proyecto de Ley de Seguridad y Convivencia Ciudadana. Quito, Ecuador: FLACSO.

Kanan, J., \& Pruitt, M. (2002). Modeling fear of crime and perceived victimization risk: the (in) significance of neighborhood integration. Sociological Inquiry, 72(4), 527-548. doi: 10.1111/1475-682X.00033.

Killias, M. (1990). Vulnerability: Towards a better understanding of a key variable in the genesis of fear of crime. Violence and Victims, 5(2), 97-108.

Latinobatómetro. (2013). Análisis Online país Ecuador. Recuperado de http://www.latinobarometro.org/latOnline.jsp.

Lee, M.(2007). Inventing Fear of Crime. Criminology and the politics of anxiety. Portland: Willan Publishing.

Loewenstein, G, F., Weber, E. U., Hsee, C. K., \& Welch, E. S. (2001). Risk as feelings. Psychological Bulletin, 12 (2), 267-286. doi: 10.1037//0033-2909.127.2.267.

Magnusson, D. (1978). Teoría de los tests. México: Trillas.

Martínez, A. R. (1995). Psicometría: Teoría de los tests psicológicos y educativos. Madrid: Síntesis.

Medrano, L. A., \& Trógolo, M. (2014). Validación de la escala de dificultades en la regulación emocional en la población universitaria de Córdova, Argentina. Universitas Psychologica, 13(4), 1345-1356. doi: http://dx.doi.org/10.11144/ Javeriana.UPSY13-4.vedr.

Miranda-Zapata, E., Riquelme-Mella, E., Cifuentes-Cid, H., \& Riquelme-Bravo, P. (2014). Análisis factorial confirmatorio de la Escala de habilidades sociales en universitarios chilenos. Revista Latinoamericana de Psicología, 46(2), 73-82. doi: 10.1016/S0120-0534(14)70010-X.

Molina-Coloma, V., Reyes-Sosa, H., \& Larrañaga, M. (2015). La representación social de la inseguridad en jóvenes universitarios ecuatorianos: el caso Ambato. Pensando Psicología, 11(18), 85-95. doi: http://dx.doi.org/10.16925/ pe.v11i18.1221.

Moore, S., \& Shepherd, J. (2007). The Elements and Prevalence of Fear. The British Journal of Criminology, 47(1), 154-162. doi: 10.1093/bjc/azl006.

Ojeda, L. (2010). Delitos en el Ecuador. En Universidad Alfredo Pérez Guerrero (Eds.), Violencia, delincuencia e inseguridad en el Ecuador (pp. 17-453). Quito, Ecuador: Universidad Alfredo Pérez Guerrero.

Reyes, H., Valencia, J., \& Larrañaga, M. (Octubre, 2015). Estructura factorial y consistencia internar de un instrumento para medir el miedo al delito en jóvenes mexicanos. XIII Congreso Mexicano de Psicología. Sociedad Mexicana de Psicología, México.
Ruiz, M.A., Pardo, A., \& San Martín, R. (2010). Modelos de ecuaciones estructurales. Papeles del Psicólogo. 3(1), 34-45.

Sampson, R., \& Stephen, R. (1999). Systematic Social Observation of Public Spaces: A New Look at Disorder in Urban Neighborhoods. American Journal of Sociology, 105(3), 603-651. doi: 10.1086/210356.

Santisteban, R. C. (2009). Principios de psicometría. Madrid: Sintesis.

Scott, H. (2003). Stranger danger: Explaining women's fear of crime. Western Criminology Review, 4(3), 203-214.

Slovic, P., Finucane, M., Peters, E., \& Macgregor, D. G. (2004). Risk as analysis and risk as feeling: Some thoughts about affects, reason, risk and rationality. Risk Analysis, 24(2), 311-322. doi: 10.1111/j.0272-4332.2004.00433.x.

Sutherland, E. (1939). Principles of Criminology. Filadelfia: Lippincott.

Steiger, J. H. (2007). Understanding the limitations of global fit assessment in structural equation modeling. Personality and Individual Differences, 42(5), 893-898. doi: https://doi. org/10.1016/j.paid.2006.09.017.

Van der Wurff, A., Van Staalduinen, L., \& Stringer, P. (1989). Fear of crime in residential enviroments: Testing a Social Model. Journal of Social Psychology, 129(2), 60-141.

Vilalta, C. J. (2009a). El miedo al crimen en México. Estructura lógica, bases empíricas y recomendaciones iniciales de política pública. Gestión y política pública, 19(1), 3-36.

Vilalta, C. J. (2009b). Los factores de la violencia durante el delito en México: armas de fuego, momento del día y experiencia criminal. Estudios Sociológicos, 37(79), 211-235.

Virla, M. (2010). Confiabilidad y coeficiente Alpha de Cronbach. Telos, 12(2), 248-252.

Vozmediano, L., San Juan, C., \& Vergara, A. (2008). Problemas de medición del miedo al delito. Algunas respuestas teóricas y técnicas. Revista Electrónica de Ciencia Penal y Criminología, 1, 7-17.

Warr, M. (2006). El miedo al delito en los Estados Unidos: Rutas para la investigación y la formulación de políticas. En R. Barberet \& J. Barquín, (Eds.), Justicia Penal Siglo XXI. Una Selección de Criminal Justice 2000.Granada: National Institute of Justice.

Winkel, F. W. (1999). Repeat victimization and trauma-susceptibility: Prospective and longitudinal analyses. En J. M. Van Dijk, R. G. H. Van Kaam \& J. A. Wemmers (Eds.), Caring for crime victims (pp. 207-221). Nueva York: Criminal Justice Press.

Williamson, T., David, A., \& Richard, W. (2006). Classifying Neighborhoods for Reassurance Policing. Policing and Society, 16(2), 189-218. doi: 10.1080/10439460600662197. 